\title{
Effects of hyperglycaemia on sorbitol and myo-inositol contents of cultured embryos: treatment with aldose reductase inhibitor and $m y o$-inositol supplementation
}

\author{
M. Hashimoto, S. Akazawa, M. Akazawa, M. Akashi, H. Yamamoto, Y. Maeda, Y. Yamaguchi, H. Yamasaki, \\ D. Tahara, T. Nakanishi and S. Nagataki \\ The First Department of Internal Medicine, Nagasaki University School of Medicine, Nagasaki, Japan
}

\begin{abstract}
Summary. To demonstrate the myo-inositol depletion hypothesis in hyperglycaemia-induced embryopathy, rat conceptuses of 9.5 days of gestation in the early head-fold stage were grown in vitro during neural tube formation for $48 \mathrm{~h}$ with increasing amounts of glucose. The effects of an aldose reductase inhibitor and the myo-inositol supplementation were also investigated. Sorbitol and myo-inositol contents were measured in separated embryos and extra-embryonic membranes including yolk sac and amnion at the end of culture. After addition of $33.3 \mathrm{mmol} / \mathrm{l}$ and $66.7 \mathrm{mmol} / 1$ glucose to the culture media, the myo-inositol content of the embryos was significantly decreased by $43.1 \% \quad(p<0.05)$ and $64.6 \%$ $(p<0.01)$ of the control group, while a marked accumulation of sorbitol was observed ( 25 and 41 times that of the control). Although the addition of an aldose reductase inhibitor $(0.7 \mathrm{mmol} / \mathrm{l})$ to the hyperglycaemic culture media containing an additional $66.7 \mathrm{mmol} / \mathrm{l}$ glucose significantly reduced the sorbitol content of embryos to approximately one-eighth, the
\end{abstract}

myo-inositol content of embryos remained decreased and the frequency of neural lesions was unchanged $(23.1 \%$ vs $23.9 \%$, NS). Supplementation of the myo-inositol $(0.28 \mathrm{mmol} / \mathrm{l})$ completely restored the $m y o$-inositol content of the embryos and resulted in a significant decrease in the frequency of neural lesions $(7.1 \%$ vs $23.9 \%, p<0.01)$ and a significant increase in crown-rump length and somite numbers. Much less significantly, sorbitol accumulation was also observed in the extra-embryonic membrane in response to hyperglycaemia, neither hyperglycaemia nor the myo-inositol supplementation modified the myo-inositol contents of the extra-embryonic membrane. We conclude that the mechanism of hyperglycaemia-induced teratogenicity was mediated by the myo-inositol depletion of the embryo at a critical stage of organogenesis.

Key words: Hyperglycaemia, embryogenesis, rat embryo culture, malformation, sorbitol, myo-inositol.
The incidence of congenital malformations in infants of diabetic mothers is three to four times greater than that found in the general neonatal population [1-3]. These malfomations, which may occur before the 7 th week of gestation include caudal regression syndrome, central nervous system deformities such as anencephaly, spina bifida and hydrocephalus, and renal and heart anomalies [4]. Although the precise aetiology of congenital malformations remains unclear, several serum factors in experimentally induced diabetes including hyperglycaemia, hypoglycaemia, hyperketonaemia and somatomedin inhibitor have been shown to elicit dysmorphogenesis in the rodent embryo culture system [5-21, 29].
Hyperglycaemia, one of the main metabolic abnormalities associated with the diabetic state, has been shown to play an important role in the teratogenesis of diabetic pregnancy. In vitro studies have demonstrated by the addition of glucose to normal serum disturbed embryonic development and malformations [6-10]. In recent studies of the pathogenesis of diabetic complications, intracellular alterations in sorbitol and myo-inositol (MI) metabolism have been regarded as important contributing factors in diabetic complications. The presence of polyol pathways has been implied even in the early stages of embryonic development, since sorbitol contents were in creased in the embryo during early organogenesis from diabetic mothers [22]. However, treatment with aldose re- 
Table 1. Effects of added glucose, aldose reductase inhibitor (ARI) and myo-inositol (MI) supplementation on sorbitol and MI contents of embryos

\begin{tabular}{|c|c|c|c|c|}
\hline $\begin{array}{l}\text { Normal culture } \\
\text { medium plus: }\end{array}$ & No. & $\begin{array}{l}\text { Protein } \\
(\mu g)\end{array}$ & $\begin{array}{l}\text { MI } \\
\text { (nmol/g } \\
\text { protein) }\end{array}$ & $\begin{array}{l}\text { Sorbitol } \\
\text { (nmol/g } \\
\text { protein) }\end{array}$ \\
\hline 0 & 15 & $213 \pm 9$ & $1612 \pm 154$ & $307 \pm 54$ \\
\hline $\begin{array}{l}16.7 \mathrm{mmol} / 1 \\
\text { D-glucose }\end{array}$ & 5 & $216 \pm 6$ & $1389 \pm 105$ & $7000 \pm 1130^{b}$ \\
\hline $\begin{array}{c}33.3 \mathrm{mmol} / \mathrm{l} \\
\text { D-glucose }\end{array}$ & 10 & $192 \pm 9$ & $918 \pm 183^{\mathrm{a}}$ & $7825 \pm 1071^{b}$ \\
\hline $\begin{array}{l}66.7 \mathrm{mmol} / \mathrm{l} \\
\text { D-glucose }\end{array}$ & 9 & $171 \pm 10^{\mathrm{a}}$ & $570 \pm 94^{b}$ & $12,584 \pm 1080^{b}$ \\
\hline $\begin{array}{l}66.7 \mathrm{mmol} / \mathrm{D}- \\
\text { glucose + ARI }\end{array}$ & 5 & $173 \pm 6^{a}$ & $628 \pm 76^{\circ}$ & $1464 \pm 475^{\mathrm{b}, \mathrm{d}}$ \\
\hline $\begin{array}{l}66.7 \mathrm{mmol} / 2 \mathrm{D}- \\
\text { glucose }+\mathrm{MI}\end{array}$ & 4 & $198 \pm 4^{\mathrm{c}}$ & $1567 \pm 245^{d}$ & $15,714 \pm 585^{b}$ \\
\hline
\end{tabular}

Rat conceptuses were explanted on day 9.5 and cultured for $48 \mathrm{~h}$ in culture media containing; $6.7 \mathrm{mmol} / \mathrm{l}$ glucose (control); additional $16.7,33.3,66.7 \mathrm{mmol} / 1$ glucose; additional $66.7 \mathrm{mmol} / \mathrm{l}$ glucose plus ARI $(0.7 \mathrm{mmol} / 1$ ONO 2300); additional $66.7 \mathrm{mmol} / \mathrm{l}$ glucose plus $\mathrm{MI}(0.28 \mathrm{mmol} / \mathrm{l} m y o$-inositol $)$. At the end of culture, embryos were separated from extra-embryonic membranes. 12-15 embryos were pooled and extracted by ethanol as shown in "Materials and methods". No. denotes the number of separate cultures performed for each condition. Protein denotes its content per embryo.

${ }^{a} p<0.05,{ }^{b} p<0.01$, significantly different from value obtained from embryos cultured with control media; ${ }^{c} p<0.05,{ }^{d} p<0.01$, significantly different from value obtained from embryos cultured with media containing additional $66.7 \mathrm{mmol} / \mathrm{l}$ glucose

Table 2. Effects of added glucose, aldose reductase inhibitor (ARI) and myo-inositol (MI) supplementation on sorbitol and MI contents of extra-embryonic membranes

\begin{tabular}{|c|c|c|c|c|}
\hline $\begin{array}{l}\text { Normal culture } \\
\text { medium plus: }\end{array}$ & No. & $\begin{array}{l}\text { Protein } \\
(\mu \mathrm{g})\end{array}$ & $\begin{array}{l}\text { MI } \\
\text { (nmol/g } \\
\text { protein) }\end{array}$ & $\begin{array}{l}\text { Sorbitol } \\
\text { (nmol/g } \\
\text { protein) }\end{array}$ \\
\hline 0 & 6 & $122 \pm 5$ & $475 \pm 92$ & $394 \pm 101$ \\
\hline $\begin{array}{l}33.3 \mathrm{mmol} / 1 \\
\text { D-glucose }\end{array}$ & 4 & $124 \pm 13$ & $515 \pm 255$ & $2628 \pm 379^{a}$ \\
\hline $\begin{array}{l}66.7 \mathrm{mmol} / \mathrm{l} \\
\text { D-glucose }\end{array}$ & 5 & $115 \pm 16$ & $367 \pm 109$ & $3351 \pm 474^{a}$ \\
\hline $\begin{array}{l}66.7 \mathrm{mmol} / / \mathrm{D}- \\
\text { glucose + ARI }\end{array}$ & 4 & $132 \pm 17$ & $276 \pm 101$ & $2107 \pm 388^{\mathrm{a}}$ \\
\hline $\begin{array}{l}66.7 \mathrm{mmol} / / \mathrm{D}- \\
\text { glucose + MI }\end{array}$ & 5 & $123 \pm 6$ & $369 \pm 44$ & $3002 \pm 534^{a}$ \\
\hline
\end{tabular}

Rat conceptuses on day 9.5 were explanted and cultured for $48 \mathrm{~h}$ as listed in Table 1 . After the culture, extra-embryonic membranes such as yolk sac and amnion were detached from embryos, 20 24 membranes were pooled and extracted by ethanol. No. denotes number of separate experiments. Protein denotes its content per extra-embryonic membrane.

a $p<0.05$, significantly different from values obtained from extraembryonic membranes cultured with control media

ductase inhibitor (ARI) did not prevent malformations in the embryos [22-24].

Impairment of MI metabolism has been proposed as being responsible for the malformations, because dietary administration of MI to diabetic rats and addition of MI to culture media protects against neural tube defects induced by hyperglycaemia [25]. Hod et al. recently showed evidence of MI depletion in the whole conceptus consisting of the embryo and extra-embryonic membrane when the conceptus was cultured in hyperglycaemic media [24].

In our study, whole conceptuses were cultured during the period of neural tube formation in the presence of hyperglycaemia. Sorbitol and MI contents were measured in the embryo as well as in extra-embryonic membranes. Furthermore, to clarify the significance of sorbitol accumulation and MI depletion during neural tube formation, the effects of ARI and MI supplementation on embryogenesis were investigated in the embryo and extraembryonic membranes.

\section{Materials and methods}

Embryo culture techniques were performed using the methods of New [5]. Virgin Wistar rats (Shizuoka Laboratory Animal Center, Shizuoka, Japan) were mated and pregnancy was timed from the preceding midnight of the morning when sperm were present in the vaginal smear. On day 9.5 of gestation when embryos were at the early head-fold stage the mothers were killed by cervical dislocation. Conceptuses were excised, floated in Petri dishes containing Hanks' balanced salt solution and freed of decidua. Reichert's membrane was opened and intact embryo units (embryos together with their visceral yolk sac, amnion and ectodermal cone) were explanted into culture media. Culture media consisted of the immediately centrifuged, heat-inactivated serum from normal female rats diluted $3: 1$ with isotonic $(0.85 \% \mathrm{NaCl})$ saline to give a final glucose concentration of $6.67 \mathrm{mmol} / \mathrm{l}$ (control) or isosmotic solution of test solute. The latter was isosmotically replaced by $5 \%$ glucose or $5 \%$ MI (Sigma Chemical Company, St. Louis, Mo., USA) in water plus $0.85 \% \mathrm{NaCl}$ to obtain a hyperglycaemic medium (containing additional $16.7 \mathrm{mmol} / 1,33.3 \mathrm{mmol} / \mathrm{l}$, or $66.7 \mathrm{mmol} / \mathrm{l}$ glucose) and control or hyperglycaemic media supplemented with MI. ARI (ONO $2235 \mathrm{~N}$ methylamine salt, Ono Company, Osaka, Japan) was dissolved in $0.85 \% \mathrm{NaCl}$ or $5 \%$ glucose. Each culture tube containing 6 embryo units in $6 \mathrm{ml}$ medium was incubated at $37^{\circ} \mathrm{C}$ with rotation in a temperature-controlled incubator. Embryo units were transferred to new culture vessels after $24 \mathrm{~h}$ and resuspended in $6 \mathrm{ml}$ of fresh culture medium for the second $24 \mathrm{~h}$ period. The embryo units were removed from the culture vessels at the end of $48 \mathrm{~h}$ on day $11.5 \mathrm{of}$ development. Embryos were separated from the yolk sac and amnion (extra-embryonic membrane) under a dissecting microscope.

Overall growth and differentiation of embryos were quantified by direct measurement of crown-rump length and somite number, respectively. Specific organs were evaluated for dysmorphogenesis by visual inspection according to a detailed check list. Malformations were divided into neural and extraneural lesions. Neural lesions indicated specific lesions involving development of neural plates such as open neural tube, open neural pore, brain anomalies and adhesion of posterior and anterior neural tube. Extraneural lesions included minor lesions such as abnormalities of axial rotation or lesions involving the optic and otic vesicles, heart size, pericardial cavity or somite system.

After visual inspection, 12-15 embryos and 20-24 pieces of extra-embryonic membranes were washed five times in $0.85 \% \mathrm{NaCl}$ and homogenized in water with microhomogenizers. After the internal standard (galactitol) was added to each sample, proteins were precipitated with ethanol to give a final concentration of $70 \%$. After centrifugation, supernatants were collected and lyophilized. The precipitates were dissolved in $0.5 \mathrm{~mol} / 1 \mathrm{NaOH}$ for the measurement of protein [26]. The lyophilized samples were derivatized with phenylisocyanate [27].

High-performance liquid chromatography, "M-45 Solvent Delivery System" (Waters Associates Inc., Mass., USA) with spectro- 
photometric detector SPD-2A (Shimazu Seisakusha Ltd., Kyoto, Japan) and a Nucleosil $5 \mathrm{C}_{18}$ liquid chromatography column (Macherey-Nagel, Düren, FRG) were used for the chromatographic separation and detection of the derivatives.

\section{Statistical analysis}

Statistical analysis was done by two-tailed Student's $t$-test for unpaired data and Wilcoxon's rank-sum test for non-parametric data. Intergroup differences for comparison of morphologic lesions were assessed by Chi-square analysis [28]. Data are expressed as mean \pm SEM.

\section{Results}

The effects of hyperglycaemia on sorbitol and MI contents of the embryo and the extra-embryonic membrane are shown in Tables 1 and 2. The accumulation of sorbitol in the separated embryos was markedly increased an average of 23 times $(p<0.01), 25$ times $(p<0.01)$ and 41 times $(p<0.01)$ of the control in the presence of an additional $16.7,33.3,66.7 \mathrm{mmol} / 1$ glucose, respectively. In inverse proportion to the marked sorbitol accumulation, MI contents of embryos progressively decreased by $43.1 \%$ $(p<0.05)$ and $64.6 \% \quad(p<0.01)$ of the control in the presence of an additional 33.3 and $66.7 \mathrm{mmol} / \mathrm{l}$ glucose, respectively (Table 1 ).

The effects of ARI and MI supplementation on the culture media containing an additional $66.7 \mathrm{mmol} / \mathrm{l}$ glucose were examined to evaluate the significance of sorbitol accumulation and MI depletion in embryos cultured under hyperglycaemic conditions. By adding ARI to hyperglycaemic culture media, sorbitol contents of the embryo dramatically decreased to approximately oneeighth $(p<0.01)$ but levels remained significantly higher than those of the control $(p<0.01)$. The significant reduction of sorbitol by ARI was not accompanied by an increase in MI content of the embryo ( $628 \pm 76$ vs $570 \pm 94 \mathrm{nmol} / \mathrm{g}$ protein, NS). By MI supplementation

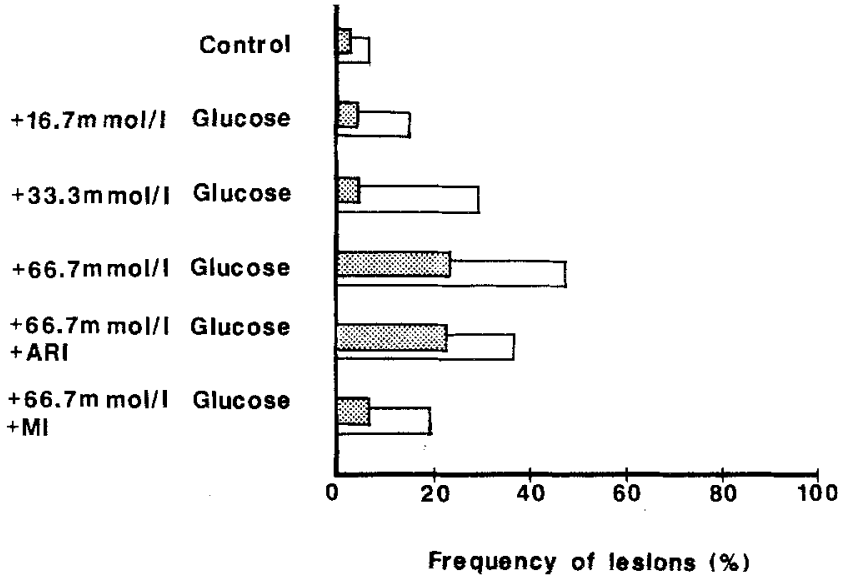

Fig. 1. Effect of added glucose, aldose reductase inhibitor and the $m y o$-inositol supplementation on frequency of malformations. Rat conceptuses were explanted on day 9.5 and cultured for $48 \mathrm{~h}$ in the presence of increasing amounts of glucose. ARI $(0.7 \mathrm{mmol} / \mathrm{L}$ ONO $2300)$ or $\mathrm{MI}(0.28 \mathrm{mmol} / \mathrm{l}$ myo-inositol) was added to hyperglycaemic media containing additional $66.7 \mathrm{mmol} / \mathrm{l}$ glucose as listed in Table 1. Length of bar indicates neural and extraneural lesions. Dotted bar indicates neural lesions and open bar, extraneural lesions. The control group consisted of 132 embryos and the effects of additional $16.7 \mathrm{mmol} / \mathrm{l}, 33.3 \mathrm{mmol} / \mathrm{l}, 66.7 \mathrm{mmol} / \mathrm{l}$ glucose and $66.7 \mathrm{mmol} / \mathrm{l}$ glucose plus ARI or MI were tested in $65,141,71,121$ and 113 embryos, respectively

$(0.28 \mathrm{mmol} / \mathrm{l})$ to the hyperglycaemic culture media, the MI contents of the embryo were significantly increased $(1567 \pm 245$ vs $570 \pm 94 \mathrm{nmol} / \mathrm{g}$ protein, $p<0.01)$ and were comparable to those of control embryos, with a persistent elevation of sorbitol (Table 1). Although sorbitol contents in the extra-embryonic membrane increased sevenfold $(p<0.05)$ and ninefold $(p<0.05)$ with the addition of 33.3 and $66.7 \mathrm{mmol} / \mathrm{l}$ glucose, the degree of increase was much less than those of the embryos. The MI contents of the extra-embryonic membrane were significantly lower $(p<0.01)$ than those of the embryos and almost half that of controls. In response to increasing amounts of glucose, the MI contents of the extra-

Table 3. Effects of added glucose, aldose reductase inhibitor (ARI) and myo-inositol (MI) supplementation on growth and development of embryo

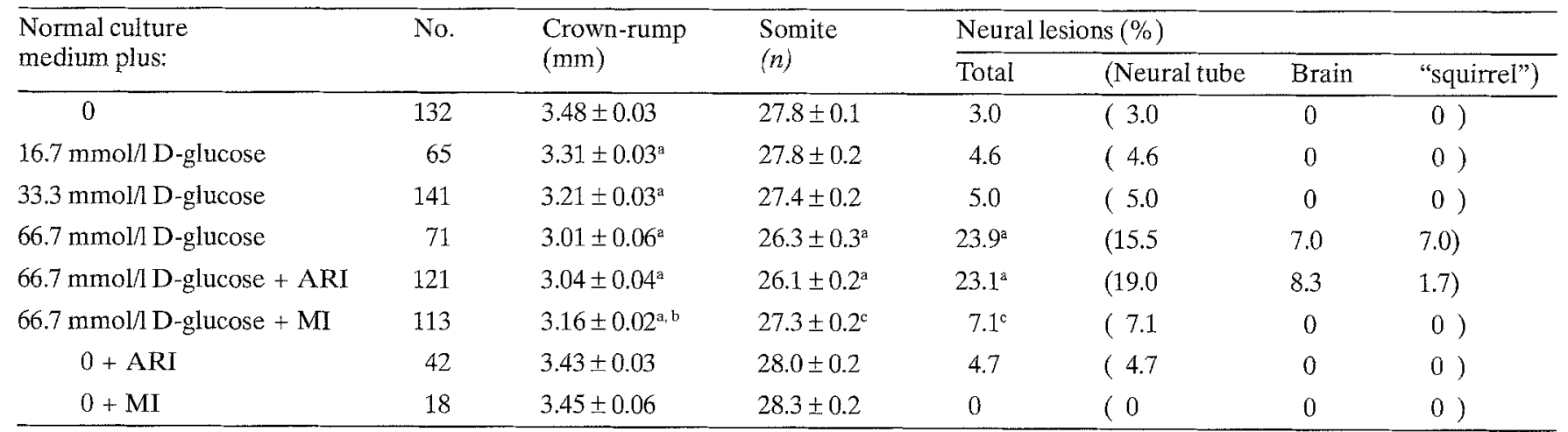

No. denote the number of embryos examined. "Total" denotes the percentage of total neural lesions consisting of defects of neural tube closure, brain anomaly and "squirrel" like lesion which is the adhesion of posterior and anterior neural tube. These lesions include multiple listings for embryos with multiple abnormalities.

${ }_{0}^{a} p<0.01$, significantly different from values obtained from embryos cultured with control media;

b $p<0.05,{ }^{c} p<0.01$, significantly different from values obtained from embryos cultured with media containing additional $66.7 \mathrm{mmol} / \mathrm{l}$ glucose 
embryonic membrane tended to decrease with the addition of $66.7 \mathrm{mmol} / \mathrm{l}$ glucose but no statistical significance was observed (Table 2).

The effect of hyperglycaemia, and ARI and MI supplementation on the frequency of malformations and growth of embryos are shown in Figure 1 and Table 3. The control serum contained 132 cultured embryos which had completely closed neural tubes, clearly delineated brains, well formed optic and otic vesicles, normal heart development and complete ventral axial rotation, except four with neural lesions (3.0\%) involving one neural tube defect and three with an open posterior neural pore, and nine with extraneural lesions $(6.8 \%)$ consisting of microencephaly and abnormalities of irregular and small somites (Fig. 1 and Table 3 ). Embryos did not show significant neural lesions (4.6\%, NS and $5.0 \%, \mathrm{NS})$ but showed extraneural lesions (15.4\%, NS and $29.1 \%$, $p<0.01$ ) when 16.7 or $33.3 \mathrm{mmol} / 1$ glucose was added to the culture media (Fig.1). The addition of $66.7 \mathrm{mmol} / 1$ glucose precipitated both neural and extraneural lesions in $23.9 \%(p<0.01)$ and $47.9 \%(p<0.01)$ of 71 embryos (Fig. 1); the neural lesions consisted of defects of the neural tube in eight $(11.3 \%)$, open posterior neuropore in three $(4.2 \%)$, anomalous brain development in five $(7.0 \%)$ and "squirrel"-like lesion which is the adhesion of the anterior and posterior neural tube in five (7.0\%) (Table 3 ). In addition to these abnormalities, the embryos showed significantly lower crown-rump length $(p<0.01)$ and somite number $(p<0.01)$ than controls (Table 3).

Supplementation of ARI to the media containing the additional $66.7 \mathrm{mmol} / \mathrm{l}$ glucose did not modify the embryonic growth (Table 3 ) or incidence of neural $(23.1 \%$ vs $23.9 \%, \mathrm{NS}$ ) and extraneural lesions ( $37.2 \%$ vs $47.9 \%$, NS) compared with those of embryos cultured with the additional $66.7 \mathrm{mmol} / 1$ glucose alone (Fig. 1), in spite of a significant reduction of sorbitol contents.

In contrast, MI supplementation to the media containing the additional $66.7 \mathrm{mmol} / \mathrm{l}$ glucose resulted in a significant decrease in the incidence of neural lesions $(7.1 \%$ vs $23.9 \%, p<0.01)$, which was comparable to those of control embryos (7.1\% vs $3.0 \%$, NS) (Fig. 1 ) and a significant increase in crown-rump length $(p<0.05)$ and somite number $(p<0.01)$, compared with those of embryos cultured with the additional $66.7 \mathrm{mmol} / \mathrm{l} \mathrm{glu}-$ cose.

\section{Discussion}

Sorbitol and MI contents of separated embryo and extraembryonic membranes were measured in the present study in hyperglycaemic culture media. The addition of glucose to culture media caused the MI content of embryos to decrease and sorbitol to accumulate in a dose-dependent fashion, while these phenomena were much less obvious in the extra-embryonic membranes. Although treatment with ARI did not modify the incidence of malformations, MI supplementation to the hyperglycaemic culture media resulted in a significantly decreased incidence of congenital malformations, with complete res- toration of MI contents of the embryos. We demonstrated that hyperglycaemia-induced teratogenesis was mediated by MI depletion of embryos at a critical stage of organogenesis.

In this study, we have also confirmed a previous report [6] of the dose-dependent teratogenic effects of hyperglycaemia in rat embryo culture. The glucose concentration of embryonic tissues approximately equals that of maternal serum [30], glucose seems to be freely permeable into embryonic tissues, probably by an insulin-independent process. Thus, hyperglycaemia caused higher concentrations of intracellular glucose with activation of the polyol pathway and lead to increased sorbitol accumulation. This phenomenon has been observed in many other tissues such as the peripheral nerves [31], lens [32], retina [33, 34] and mesangial cells [35]. The excessive accumulation of this polyol predominantly in embryonic tissues suggests that aldose reductase (AR) is already present in embryos during early organogenesis and readily inducible by a diabetic environment. Sorbitol accumulation has been reported in embryos from experimental diabetes in vivo [22] and in the whole conceptus which was cultured with high glucose in vitro [24]. We observed a much greater sorbitol accumulation in the separated embryos than in whole conceptuses cultured under similar conditions, indicating that the rise in sorbitol in the whole conceptus was obtunded by the mixture of structurally different tissues which consisted of embryo and extra-embryonic membranes. This accumulation does not appear to be a major factor in the early developmental disturbance of embryos cultured under hyperglycaemic conditions. Treatment with ARI did not modify the incidence of malformations and abnormalities of embryonic development, in spite of a significant reduction of sorbitol in the embryo. Our findings confirmed previous reports that the administration of ARI (Statil) to pregnant diabetic rats in vivo [22] and the addition of ARI $(50 \mu \mathrm{mol} / 1$ Sorbinil or Statil) to hyperglycaemic culture media in vitro [24] did not modify the overall incidence of malformations. An increase of sorbitol is consistently associated with a fall in MI content in most tissues including the retina [34], nerve [36] and kidney [37] and both phenomena are usually reversed by ARI $[34,36,37]$.

In the present study, graded hyperglycaemia was attended by dose-dependent sorbitol accumulation and stepwise MI depression in embryonic tissue. However, a significant reduction of sorbitol accumulation in the embryo by ARI administration was not accompanied by an elevation of MI. Since administration of MI or arachidonic acid can protect against neural tube defects, it has been hypothesized that impaired MI homeostasis has an important role in diabetic embryopathy $[25,38,39]$. We have measured MI contents in separated embryo and membranes, and have shown they are progressively diminished by increasing hyperglycaemia in the separated embryo. They are not affected, however, in the extra-embryonic membrane by the presence of hyperglycaemia or MI supplementation. Therefore, MI depression appears to be a specific phenomenon in embryonic tissues, leading to defects of neural tube closure. 
In order to completely restore MI depression of embryos in this study, $0.28 \mathrm{mmol} / \mathrm{l} \mathrm{MI}$ at a concentration approximately tenfold higher than in normal rat serum, was required to be added to the hyperglycaemic culture media, which contained a tenfold higher concentration of glucose. Since labelled MI uptake [40] or MI utilization in the embryo was diminished in a dose-dependent manner in the presence of added glucose (data not shown), MI depression of embryonic tissue may occur from competition with ambient glucose for uptake rather than diminished generation from intracellular precursors. The importance of MI depletion in the pathogenesis of hyperglycaemia-induced malformations has been described. We also observed that MI contents were significantly decreased in embryos during early organogenesis from streptozotocininduced diabetic mothers. Although MI depletion was completely restored both by treatment with insulin and by oral administration of MI, the latter only partially protected against malformations induced by diabetes, while the former did so completely (unpublished data). These results indicate that MI deficiency of embryos at a critical stage of organogenesis also contributes to the pathogenesis of diabetes-induced malformations. However, whether or not depletion of MI leads to diminished generation of the key phosphoinositide which plays a critical role in signal transduction, remains to be resolved.

Acknowledgements. The authors are grateful to Dr. K. Shimaoka, Chief of Nagasaki Laboratory, Radiation Effects Research Foundation for reviewing this manuscript. We acknowledge the expert technical assistance of M. Nishimura.

\section{References}

1. Mills JL (1982) Malformations in infants of diabetic mothers. Teratology 25: 385-394

2. Small M, Cassidy L, Leiper JM, Paterson KR, Lunan CB, Mac Cuish AC (1986) Outcome of pregnancy in insulin-dependent (Type I) diabetic women between 1971 and 1984. Q J Med 61: 1159-1169

3. Nelson RL (1986) Diabetes and pregnancy: control can make a difference. Mayo Clin Proc 61: 825-829

4. Mills JL, Baker L, Goldman AS (1979) Malformations in infants of diabetic mothers occur before the seventh gestational week. Implications for treatment. Diabetes 28: 292-293

5. New DAT (1978) Whole embryo culture and the study of mammalian embryos during organogenesis. Biol Rev 53: 81-122

6. Freinkel N, Cockroft DL, Lewis NJ, Gorman L, Akazawa S, Phillips LS, Shambaugh GE (1986) The 1986 McCollum award lecture. Fuel-mediated teratogenesis during early organogenesis: the effects of increased concentrations of glucose, ketones, or somatomedin inhibitor during rat embryo culture. Am J Clin Nutr 44: 986-995

7. Sadler TW (1980) Effects of maternal diabetes on early embryogenesis. II. Hyperglycemia-induced exencephaly. Teratology 21:349-356

8. Cockroft DL, Coppola PT (1977) Teratogenic effects of excess glucose on head-fold rat embryos in culture. Teratology 16:141146

9. Sadler TW (1980) Effects of maternal diabetes on early embryogenesis. I. The teratogenic potential of diabetic serum. Teratology 21: 339-347

10. Garnham EA, Beck F, Clarke CA, Stainsstreet M (1983) Effects of glucose on rat embryos in culture. Diabetologia 25:291-295
11. Ellington SKL (1987) Development of rat embryos cultured in glucose-deficient media. Diabetes 36: 1372-1378

12. Akazawa S, Akazawa M, Hashimoto M, Yamaguchi Y, Kuriya N, Toyama K, Ueda Y, Nakanishi T, Mori T, Miyake S, Nagataki S (1987) Effects of hypoglycaemia on early embryogenesis in rat embryo organ culture. Diabetologia 30: 791-796

13. Horton WE, Sadler TW (1983) Effects of maternal diabetes on early embryogenesis. Alterations in morphogenesis produced by the ketone body, B-hydroxybutyrate. Diabetes 32: 610-616

14. Sadler TW, Phillips LS, Balkan W, Goldstein S (1986) Somatomedin inhibitors from diabetic rat serum alter growth and development of mouse embryos in culture. Diabetes 35: 861-865

15. Shepard TH, Tanimura T, Robkin MA (1970) Energy metabolism in early mammalian embryos. Symp Soc Dev Biol 29: 42-58

16. Freinkel N, Lewis NJ, Akazawa S, Gorman L, Potaczek M (1983) The honeybee syndrome: teratogenic effects of mannose during organogenesis in rat embryo culture. Trans Assoc Am Physicians 96: 44-55

17. Freinkel N, Lewis NJ, Akazawa S, Roth SI, Gorman L (1984) The honeybee syndrome-implications of the teratogenicity of mannose in rat-embryo culture. N Engl J Med 310: 223-230

18. Lewis NJ, Akazawa S, Freinkel N (1983) Teratogenesis from $\beta$ hydroxybutyrate during organogenesis in rat embryo organ culture and enhancement by subteratogenic glucose. Diabetes 32 [Suppl 1]: 11A

19. Sheehan EA, Beck F, Clarke CA, Stanisstreet M (1985) Effects of $\beta$-hydroxybutyrate on rat embryos grown in culture. Experientia 41:273-275

20. Sadler TW, Hunter ES III, Wynn RE, Phillips LS (1989) Evidence for multifactorial origin of diabetes-induced embryopathies. Diabetes 38: 70-74

21. Hunter ES III, Sadler TW, Wynn RE (1987) A potential mechanism of DL- $\beta$-hydroxybutyrate-induced malformations in mouse embryos. Am J Physiol 235: 72-80

22. Eriksson UJ, Naeser P, Brolin SE (1986) Increased accumulation of sorbitol in offspring of manifest diabetic rats. Diabetes 35 : 1356-1363

23. Eriksson UJ, Brolin SE, Naeser P (1989) Influence of sorbitol accumulation on growth and development of embryos cultured in elevated levels of glucose and fructose. Diab Res 11:27-32

24. Hod M, Star S, Passonneau JV, Unterman TG, Freinkel N (1986) Effect of hyperglycemia on sorbitol and myo-inositol content of cultured rat conceptus: failure of aldose reductase inhibitors to modify myo-inositol depletion and dysmorphogenesis. Biochem Biophys Res Comm 140: 974-980

25. Baker L, Piddington R, Goldman AS, Dahlem S, Egler J (1986) Myoinositol (MI) and Arachidonic acid (AA) are linked in the mechanism of diabetic embryopathy. Diabetes 35 [Suppl 1]: $12 \mathrm{~A}$

26. Lowry OH, Rosenbrough NJ, Farr AL, Randall RJ (1951) Protein measurement with the Folin Phenol reagent. J Biol Chem 193:265-275

27. Dethy JM, Callaert-Deveen B, Janssens M, Lenaers A (1984) Determination of sorbitol and galactitol at the nanogram level in biological samples by high-performance liquid chromatography. Anal Biochem 143: 119-124

28. Snedecor GW, Cochran WG (1980) Statistical Methods, 7th edn. Iowa State University Press, Ames, Iowa, pp 64-130

29. Sadler TW, Hunter ES III (1987) Hypoglycemia: how little is too much for the embryo? Am J Obstet Gynecol 157: 190-193

30. Sussman I, Matschinsky FM (1988) Diabetes affects sorbitol and myo-inositol levels of neuroectodermal tissue during embryogenesis in rat. Diabetes 37: 974-981

31. Gabbay KH (1973) The sorbitol pathway and the complications of diabetes. N Engl J Med 288: 831-836

32. Yeh LA, Rafford CE, Beyer TA, Hutson NJ (1986) Effects of the aldose reductase inhibitor sorbinil on the isolated cultured rat lens. Metabolism 35 [Suppl 1]: 4-9

33. MacGregor LC, Matschisky FM (1986) Experimental diabetes mellitus impairs the function of the retinal pigmented epithelium. Metabolism 35 [Suppl 1]: 28-34 
34. Williamson JR, Chang K, Rowold E, Marvel J, Tomlinson M, Sherman WR, Ackerman KE, Kilo C (1986) Diabetes-induced increases in vascular permeability and change in granulation tissue levels of sorbitol, myo-inositol, chiro-inositol, and scylloinositol are prevented by sorbinil. Metabolism 35 [Suppl 1]: 4145

35. Kikkawa R, Umemura K, Haneda M, Arimura T, Ebata K, Shigeta Y (1987) Evidence for existence of polyol pathway in cultured rat mesangial cells. Diabetes $36: 240-243$

36. Finegold D, Lattimer SA, Nolle S, Bernstein M, Green DA (1983) Polyol pathway activity and myo-inositol metabolism. A suggested relationship in the pathogenesis of diabetic neuropathy. Diabetes 32: 988-992

37. Cohen MP (1986) Aldose reductase, glomerular metabolism, and diabetic nephropathy. Metabolism 35 [Suppl 1]:55-59

38. Pinter E, Reece EA, Leranth CZ (1986) Arachidonic acid prevents hyperglycemia-associated yolk sac damage and embryopathy. Am J Obstet Gynecol 155: 691-702
39. Goldman AS, Baker L, Piddington R, Marx B, Herold R, Egler J (1985) Hyperglycemia-induced teratogenesis is mediated by a functional deficiency of arachidonic acid. Proc Natl Acad Sci USA 82: 8227-8231

40. Weigensberg M, Garcia-Palmer F, Freinkel N (1987) Competition between glucose and myo-inositol for transport in the embryo: a possible contributor to the embryopathy of hyperglycemia? Clin Res 35: 863A

Received: 18 December 1989

and in revised form: 30 April 1990

Dr. S. Nagataki

The First Department of Internal Medicine

Nagasaki University School of Medicine

Nagasaki 852

Japan 